\title{
UN ENCLAVE SOLUTRENSE EN LAS CORDILLERAS BÉTICAS OCCIDENTALES: LA CUEVA DEL HIGUERAL- GUARDIA (CORTES DE LA FRONTERA, MÁLAGA, ESPAÑA)
}

\author{
A solutrean site in the Western Betic Range: El Higueral- \\ Guardia cave (Cortes de la Frontera, Málaga, Spain)
}

\section{Concepción Torres Navas ${ }^{1}$, Javier Baena Preysler ${ }^{1}$, Antonio Morgado Rodríguez ${ }^{1}$, José A. Lozano Rodríguez ${ }^{2}$ y Manuel Alcaraz Castaño ${ }^{3}$}

Recibido el 14 de marzo de 2013. Aceptado el 11 de julio de 2013

Resumen. En el verano de 2011 se iniciaron trabajos de sondeo en la cueva del Higueral-Guardia (Málaga, España). Los sondeos han determinado la existencia de una importante secuencia con niveles del Paleolítico superior y medio todavía en estudio. No obstante, la actividad perpetrada por los clandestinos en la cueva, ha limitado significativamente las posibilidades de interpretación de los niveles solutrenses. En este trabajo pretendemos, además de realizar una presentación preliminar de los datos obtenidos, establecer una discusión sobre el valor que este tipo de registros puede tener de cara a establecer interpretaciones de rango mayor. Aspectos como la intensidad de la ocupación, la diacronía del registro, o la funcionalidad del sitio son discutidos en este sentido.

Palabras clave: Solutrense, Musteriense, clandestinos, secuencia, tecnología lítica, sur de España.

Abstract. In the summer of 2011 several test pits were made in the Higueral-Guardia Cave (Málaga, Spain). The sondages have determined the existence of an important Upper and Middle Paleolithic archaeological sequence, still under study. However, the illegal digging activity in the cave have significantly limited the possibilities of interpretation of the Solutrean levels. In this paper we present some preliminary results of the field work, and at the same time, establish a discussion about the value of such records in order to establish deeper anthropological interpretations. Aspects such as the intensity of the occupation, the diachronic value of the archaeological record, or the functionality of the site are discussed.

Keywords: Solutrean, Mousterian, furtives, sequence, lithic technology, Southern Spain.

(1) Departamento de Prehistoria y Arqueologia. Universidad Autónoma de Madrid. Madrid (España).

(2) Departamento de Prehistoria y Arqueología. Universidad de Granada. Granada (España).

(3) Área de Prehistoria. Facultad de Filosofía y Letras. Universidad de Alcalá. Calle Colegios 2. E-28801 Alcalá de Henares (Madrid, España). manuel.alcaraz@uah.es. 


\section{INTRODUCCIÓN}

En el sur de la Península lbérica el registro arqueológico no está exento de carencias en lo que se refiere a los periodos más antiguos de la Prehistoria. Se trata de insuficiencias que se relacionan, por un lado, con estratigrafías excavadas tiempo atrás que carecen de estudios actualizados rigurosos, y por otro, con lagunas derivadas de la escasez de excavaciones y publicaciones recientes referidas a este periodo. En este contexto, el estudio secuencial de la cueva del Higueral-Guardia viene a aminorar las insuficiencias que ofrece el registro arqueológico desde el análisis de la interacción de las comunidades prehistóricas en el marco dinámico de los diferentes cambios paleoecológicos del Pleistoceno y el Holoceno antiguo-medio.

En este ámbito, concretamente entre los límites administrativos de las provincias andaluzas de Málaga y Cádiz en el denominado Cerro de las Motillas de la Serranía de Ronda, se enclava a más de $400 \mathrm{~m}$ sobre el nivel del mar (s.n.m.), la cueva del Higueral-Guardia. La cavidad se sitúa en el Parque Natural de Los Alcornocales, entre los términos municipales de Cortes de la Frontera (Málaga) y Jerez de la
Frontera (Cádiz), en el sur de España (Fig. 1). Esta región andaluza posee un patrimonio histórico que indica claramente el importante papel que esta área geográfica debió jugar en las dinámicas ocupacionales de las comunidades prehistóricas.

En términos geológicos, la cueva del Higueral-Guardia se enmarca en un complejo kárstico con diferentes cavidades y abrigos rocosos que vienen siendo objeto de estudio para el análisis de las primeras ocupaciones humanas en el contexto de la evolución paleoecológica y climática del Cuaternario de las Béticas occidentales. Un estudio que se ejecuta a partir de las actuaciones que recoge el denominado proyecto KURETES. Primeras ocupaciones humanas, evolución paleoecológica y climática del Cuaternario de las Béticas occidentales (Serranía de Ronda) (Baena et al. en prensa).

Este proyecto de investigación trata de reconstruir diacrónica y sincrónicamente los modos de vida de los primeros pobladores de esta comarca montañosa, los modelos de aprovechamiento de los recursos puestos en juego, así como el impacto ambiental que todo ello ha podido generar a lo largo del tiempo.

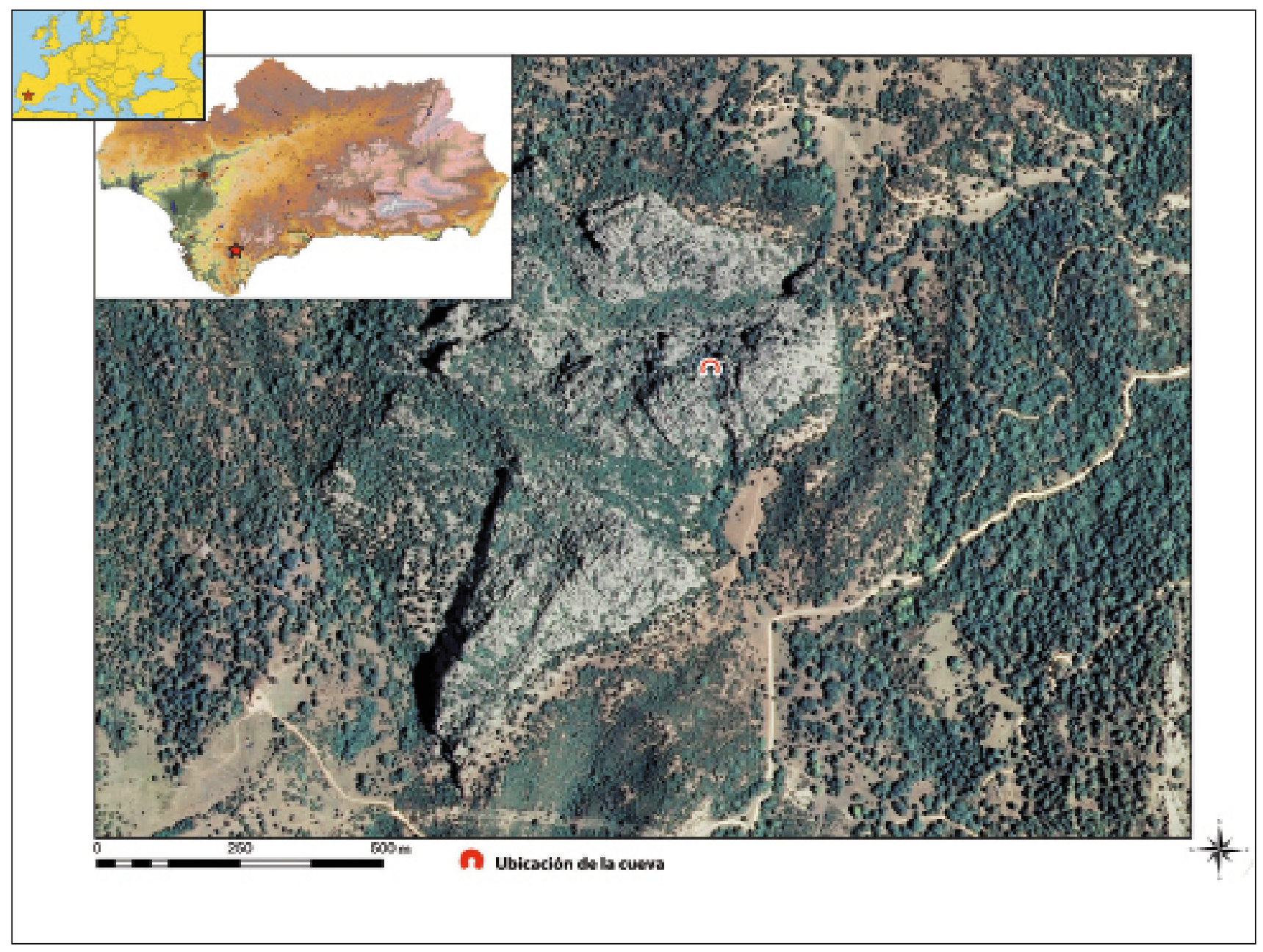

\ Figura 1. Mapa de localización de la cueva del Higueral-Guardia (cortesía de David García). 


\section{EL SOLUTRENSE EVOLUCIONADO DE LA REGIÓN SUR MEDITERRÁNEA EN LA PENÍNSULA IBÉRICA}

Todo parece indicar que durante los últimos momentos del Pleistoceno superior el territorio de las Béticas occidentales fue frecuentado por bandas de cazadores y recolectores del Paleolítico superior. Esta etapa es, sin duda, una de las que más resultados deberán ofrecer en el futuro, a tenor del alto potencial que pueden contener las secuencias estratigráficas de las cavidades de este marco territorial. Así lo han mostrado algunas actuaciones puntuales, desarrolladas para evaluar las agresiones que estas cavidades han sufrido (Giles et al. 1997, 1998). La falta de documentación sobre estos momentos no obedece a hipotéticos despoblamientos, como se ha afirmado para otras áreas de la provincia de Málaga (Ramos Muñoz 1998), paralela a planteamientos similares de la depresión del Guadalquivir (Vallespí Pérez 1992). Con ello se ha querido restringir la presencia de estos grupos cazadores y recolectores a lugares concretos, como la bahía de Málaga, con un cierto carácter de "acantonamiento". En contraposición a las hipótesis que limitan a estos grupos al ámbito litoral, encontramos emplazamientos que atestiguan la presencia solutrense en el interior de Málaga: las cueva de Ardales y de la Pileta (Cantalejo et al., 2006; Cortés y Simón 2007), sin que por el momento podamos establecer el carácter de estas ocupaciones, y con ello la estacionalidad y funcionalidad de las mismas.

Tales hipótesis son el lógico resultado de una falta de investigación sobre estos grupos en estos marcos geográficos. La primera intervención arqueológica llevada a cabo en agosto de 2011 en la cueva del Higueral - Guardia ha confirmado la existencia de una importante ocupación solutrense en esta región. Esta primera campaña consistió en la apertura de tres sondeos arqueológicos $(1,2$ y 3$)$ con la finalidad de complementar la prospección realizada previamente, reconocer la estratigrafía del yacimiento y valorar los restos arqueológicos. Al inicio de la excavación del sondeo 2 se hallaron los primeros registros solutrenses (Fig. 2) en un nivel totalmente removido y alterado (Baena 2011).

Se trataba de un nivel cuya textura suelta en el sedimento además de la intrusión de elementos recientes como plásticos o fragmentos de goma quemados indicaban que los primeros niveles excavados se encontraban removidos a causa de una previa y desconocida acción expoliadora de clandestinos en la cavidad. Aunque los niveles arqueológicos han perdido en su casi totalidad valor científico, el hallazgo de estas piezas diagnósticas atribuibles al Solutrense superior evolucionado nos invitan a valorar el potencial arqueológico de la cueva del Higueral-Guardia en un periodo que vendría caracterizado por la generalización de la punta de pedúnculo y aletas, que ya aparece en bajas proporciones en el Solutrense medio de Parpalló (a veces se le llama punta de Parpalló).

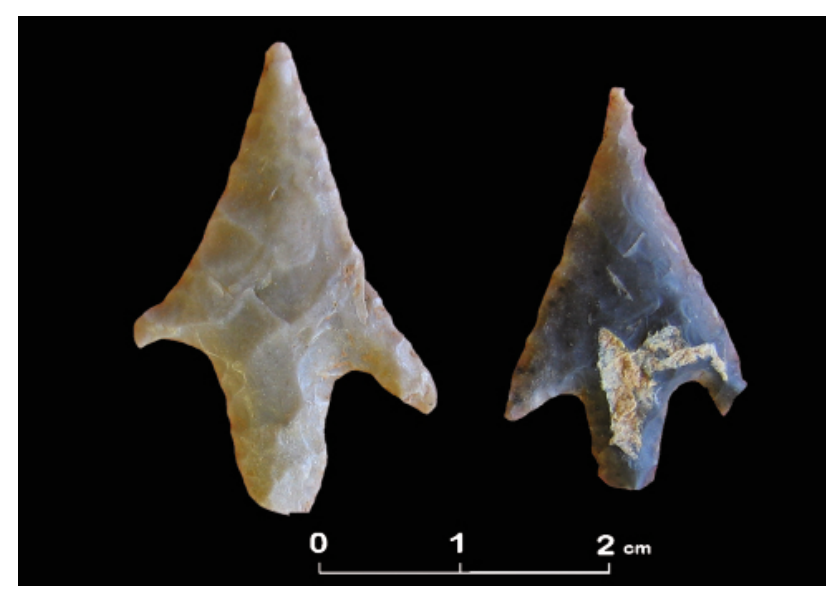

$\Delta$ Figura 2. Puntas de pedúnculo y aletas de los niveles 2-3 solutrenses. Obsérvese la asimetría en las dimensiones y profundidad de las aletas de la puntas.

El Solutrense evolucionado de la región sur mediterránea en la Península lbérica supone un momento de intensificación en la ocupación del territorio por parte de los grupos de cazadores-recolectores del Ilamado Solutrense de facies ibérica, tal como atestigua el aumento exponencial de niveles atribuidos a este tecnocomplejo en comparación con el Solutrense inferior y medio (Fullola et al. 2005; Tiffagom 2006; Tiffagom et al. 2007; Cortés et al. 2011) (Fig. 3).

De forma paralela a dicho aumento en la ocupación del territorio, la población humana parece extenderse desde las tradicionales zonas costeras, que siguen ocupándose, hacia las regiones interiores. Así, mientras que en el interior de la Andalucía oriental las primeras fases del Solutrense se encuentran representadas únicamente en el yacimiento almeriense de Ambrosio (Vélez-Blanco, Almeria), con un nivel atribuido al Solutrense medio (Ripoll 1988), y probablemente en la malagueña cueva de la Pileta (Benaoján), que alberga representaciones gráficas asignadas al Solutrense (Sanchidrián 1997) y donde se localizó una punta de cara plana (Cortés y Simón 2007: 56), durante el Solutrense superior las evidencias son algo más numerosas (Fig. 3). Sintetizamos a continuación las principales evidencias referidas a estas cronologías en los territorios interiores.

En la provincia de Cádiz contamos con datos que apuntan hacia una ocupación durante fases avanzadas del Solutrense en Higueral de Valleja (Arcos de la Frontera) (Giles et al. 1997; Jennings et al. 2009). En Málaga, al problemático lote industrial de la cueva de las Vacas o Tajo de Jorox (Alozaina) (Marqués y Ruiz 1976), ha de sumársele el nivel 1 de la cueva del Boquete de Zafarraya (Alcaucín), atribuido a una fase final del Solutrense (Barroso et al. 2006: 1597). En Granada se ha indicado la existencia de Solutreogravetiense en el yacimiento al aire libre de Pantano de Cubillas (Albolote) (Toro y Ramos Linaza 1985). Aún más al norte, en Córdoba, en el yacimiento de Pirulejo 


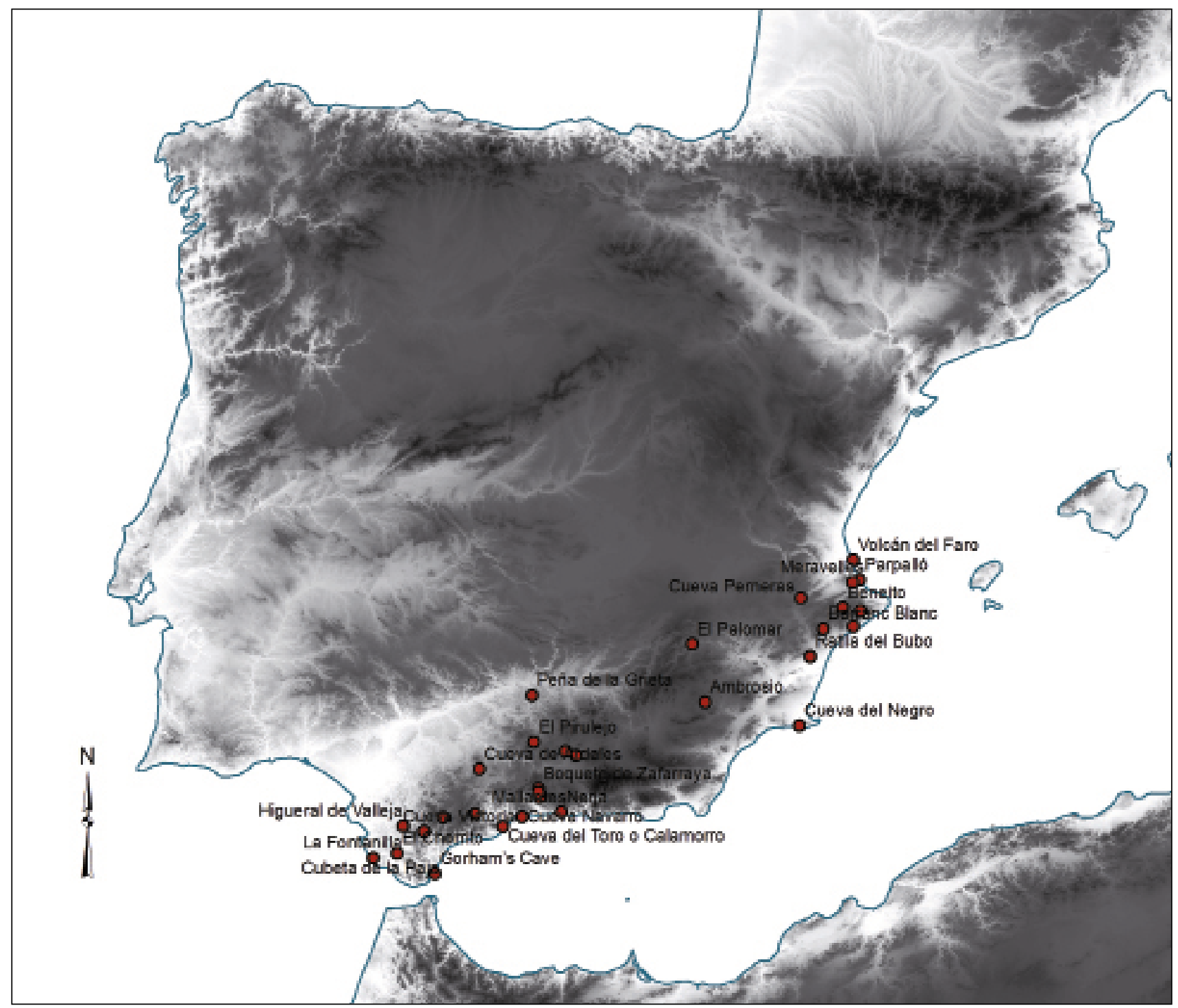

A FiguRA 3. Localización de los principales yacimientos solutrenses del sur y sureste peninsular.

encontramos un nivel con puntas de aletas y pedúnculo asignado al Solutrense superior (Cortés 2008). En Jaén contamos con el abrigo de Peña de la Grieta (Porcuna), donde se ha señalado la presencia de Solutrense superior y Solutreogravetiense (Arteaga et al. 1998). En Murcia recientemente se han revelado niveles solutreogravetienses en los abrigos de Finca de Doña Martina y La Boja (Mula) (Zilhão et al. 2010). Por último, ya en la provincia de Albacete, en la cordillera Prebética encontramos también evidencias de Solutrense evolucionado en el abrigo de El Palomar (Yeste) (Córdoba y Vega 1988).

Junto a estas evidencias de Solutrense evolucionado en las regiones interiores de la franja mediterránea meridional, es necesario valorar igualmente la presencia de representaciones gráficas solutrenses en yacimientos interiores, como el mencionado de La Pileta, el malagueño de la cueva de Ardales (Ardales) (Cantalejo et al. 2006), y el granadino de la cueva de Malalmuerzo (Moclín) (Cantalejo 1983) en los que las representaciones de marcado estilo solutrense se enmarcan en un amplio decurso gráfico que muy probablemente incluya las fases finales del tecnocomplejo (Villaverde 1994; Villaverde et al. 2009).

En este contexto, la puesta al descubierto en Higueral-Guardia de un conjunto industrial dominado por las puntas de pedúnculo y aletas viene a engrosar la lista de enclaves que atestiguan la presencia de grupos humanos en las tierras interiores del Sureste peninsular durante el Solutrense superior o evolucionado, incidiendo así en la diversificación de la ocupación del territorio que parece documentarse a partir de momentos avanzados del Solutrense. Aunque los niveles arqueológicos han perdido gran parte de su valor científico, el hallazgo de estas piezas diagnósticas atribuibles al Solutrense superior evolucionado nos invitan a valorar el potencial que este tipo de registros alterados puede tener en la investigación del $\mathrm{Pa}-$ leolítico peninsular. 


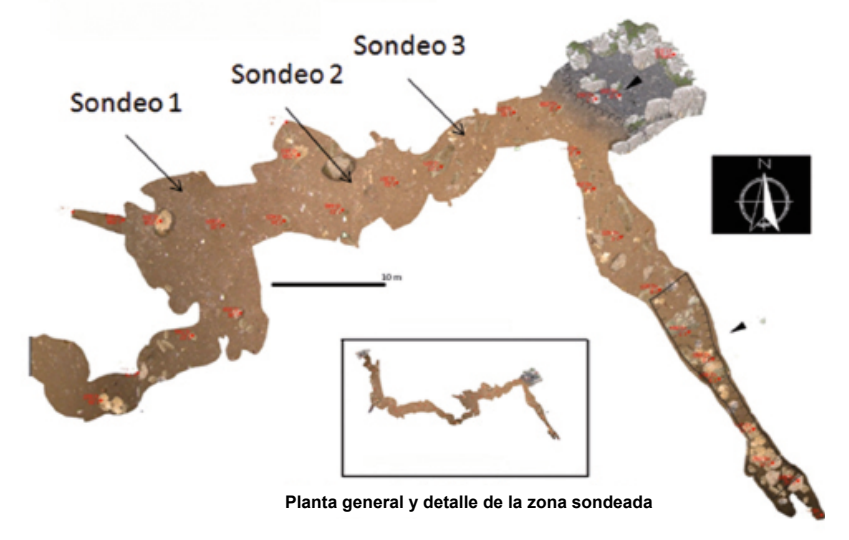

\ Figura 4. Mapa de distribución de sondeos en planta.

\section{LA CUEVA DEL HIGUERAL-GUARDIA: LAS EVIDENCIAS SOLUTRENSES QUE QUEDARON}

En la actualidad, el yacimiento de la cueva del HigueralGuardia se encuentra en fase de estudio. Pendiente aún de las aportaciones de investigadores y especialistas que integran el Proyecto Kuretes (geoarqueólogos, arqueozoólogos, antracólogos, tafónomos, palinólogos...). De modo que la primera aproximación al yacimiento se ha realizado a partir del análisis y estudio de los materiales líticos relacionados con los niveles solutrenses de los que ya podemos ofrecer los primeros resultados.

Fueron tres los sondeos realizados en la planta de la cueva (Fig. 4). En el denominado sondeo 2 se halló un significativo y diagnóstico material perteneciente al Solutrense superior evolucionado. El sondeo fue realizado debajo de una gran costra pétrea de aproximadamente $6 \mathrm{~m}^{2}$. Tras la rotura de dicha costra se iniciaron los trabajos de excavación y la secuencia documentada hasta el momento ha confirmado un nivel de ocupación solutrense profundamente afectado. Existe constancia de la realizaron de distintos agujeros que llegaron a remover cerca del $95 \%$ de la totalidad de niveles solutrenses, hecho que no hace posible discriminar dichos niveles estratigráficamente (se encontraron restos de bolsas de plástico, fragmentos quemados de caucho. etc.).

La secuencia continuó siendo excavada (hasta un número de 9 niveles arqueológicos diferenciados) poniendo de manifiesto la presencia de niveles correspondientes al Musteriense (Fig. 5). Estos niveles no han sido afectados por actividades ajenas a las arqueológicas, y estimamos que la secuencia continuará siendo excavada los próximos años.

La causa de que aparezcan niveles solutrenses removidos hemos intentado buscarla partiendo de dos hipótesis. Por una lado, se ha constatado que el guano de murciélago es uno de los fertilizantes naturales más populares utilizado desde hace cientos de años por los agricultores y en la cueva del Higueral-Guardia tenemos documentada una intensa explotación de los excrementos de quirópteros, una práctica que implica la extracción no solo de la murcielaguina sino de los sedimentos de dichas cuevas, destruyendo y alterando parte del registro arqueológico. Por otra parte, consideramos que a esta actividad se han podido sumar diferentes actuaciones clandestinas dirigidas a extraer material arqueológico significativo. De la misma manera, a partir de la segunda mitad de la década de los setenta del siglo pasado, las diferentes cavidades del Cerro de las Motillas fueron objetivo de actividades de exploración subterránea. Entre ellas destacan las Ilevadas a cabo por el Grupo de Investigaciones Espeleológicas de Jerez (GIEX), de lo cual algunas publicaciones han visto la luz, dando a conocer principalmente la presencia de manifestaciones rupestres paleolíticas (GIEX 1980; Santiago Vilchez 1980a, 1980b, 1983, 1990). Fruto de esta actividad deportiva y exploratoria fue la documentación de restos arqueológicos en algunas cavidades. Actualmente algunos de estos materiales arqueológicos, se encuentra dispersos en colecciones privadas $y$, en menor medida, en los fondos de algunos museos (Museo de Jerez y Museo de Cádiz). Los hallazgos arqueológicos no se limitan al interior de las cavidades, sino que existe toda una serie de referencias sobre el hallazgo de materiales arqueológicos fechados en el Achelense superior o "premusteriense" a lo largo de todo el cerro



$\Delta$ Figura 5. Interpretación de la secuencia del sondeo 2 de la cueva del Higueral-Guardia. 


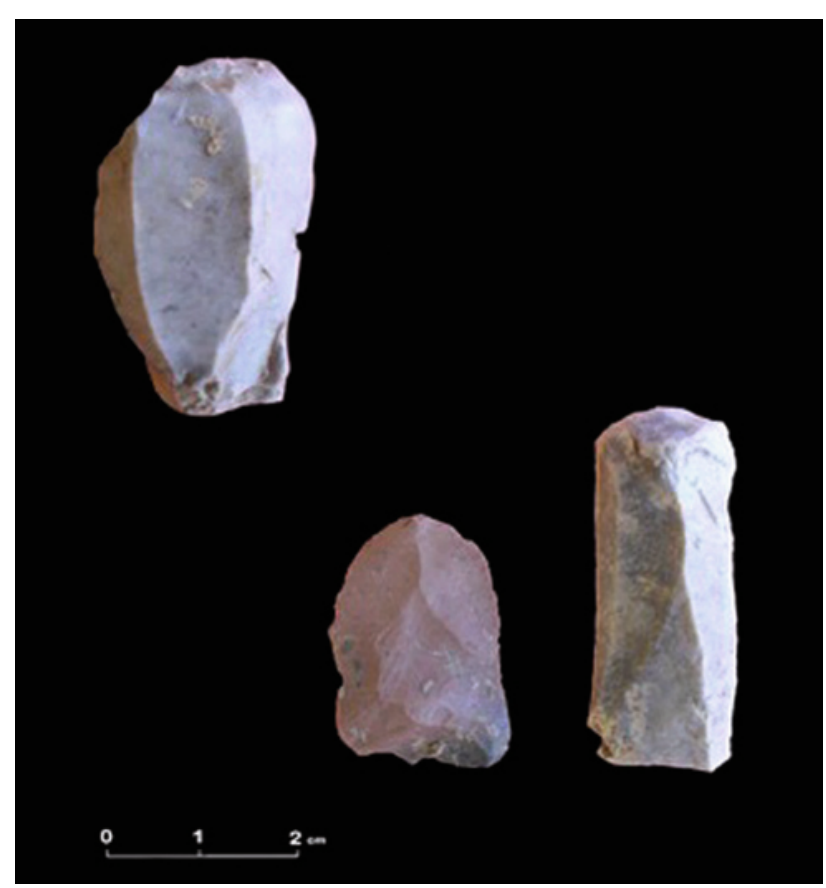

A Figura 6. Parte del material hallado en los niveles solutrenses del sondeo 2 .

de Las Motillas, sin que se tenga constancia de una ubicación exacta de estos hallazgos (Santiago Vílchez 1983; Giles et al. 2000a y 2000b).

El material hallado en los niveles solutrenses del sondeo 2 son claramente caracteristicos del Solutrense superior evolucionado andaluz. En concreto hallamos productos laminares, productos foliáceos, raspadores, núcleos laminares muy explotados, buriles, y un conjunto de puntas de pedúnculo y aletas (Fig. 6).

Realizado un estudio tecnológico preliminar del material lítico hallado, se han contabilizado un total de 1.468 piezas en los niveles solutrense. El rasgo más destacable de este conjunto industrial es la elevada proporción de elementos líticos relacionados con la producción de elementos foliáceos. Se han documentado igualmente lascas de diversa tipología, producción laminar y elementos alterados térmicamente.

Es evidente la elevada proporción de elementos líticos relacionados con restos de talla de pocos milímetros que indican que nos encontramos ante un conjunto industrial realizado básicamente sobre lascas. Por el contrario, las piezas retocadas no superan la treintena, lo que supone menos de un 5\% de la industria frente a más del 95\% de piezas que componen el total (Fig. 7). Unos porcentajes que parecen ajustarse a parámetros normales, pues se asemejan a las proporciones que muestran las evidencias solutrenses de yacimientos como Ambrosio (Almería) (Ripoll 1988) durante el mismo periodo cronocultural y en el mismo ámbito geográfico.

La habitabilidad de la cueva en momentos avanzados del Solutrense es un hecho contrastado geoarqueológicamente como lo es también la existencia de numerosas

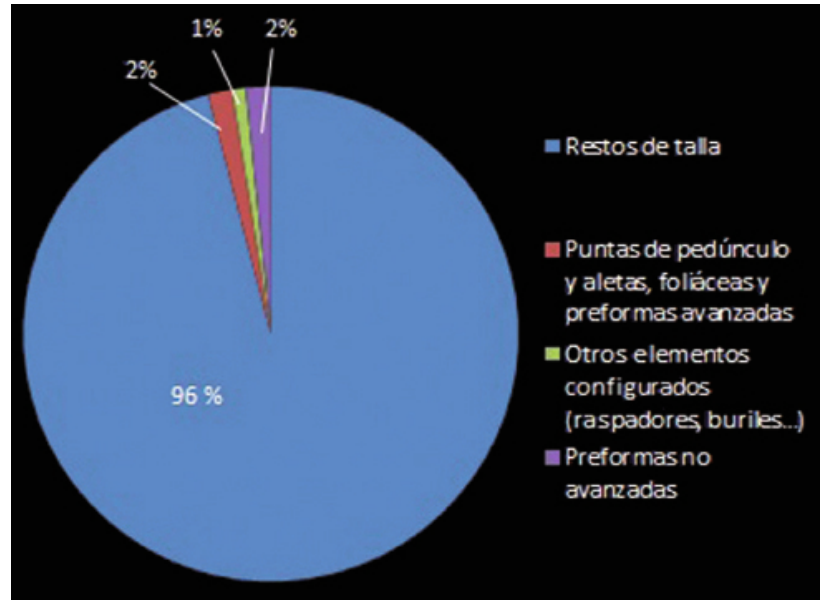

A Figura 7. Porcentaje de productos y restos de talla en los niveles solutrenses del sondeo 2 .

fuentes de materia prima de calidades diversas en las proximidades de la cueva del Higueral-Guardia. La elevada proporción de restos de talla conduce a intuir que la cavidad fue utilizada como lugar donde se elaboró el utillaje solutrense. Los útiles líticos encontrados fueron por tanto, tallados in situ. De ahí que al alto porcentaje de restos de talla se añada el hecho de que encontremos piezas en diferentes estadios de realización clasificadas según las categorías propuestas por Zilhão (1997): "piezas acabadas" con retoque plano bifacial en bandas paralelas; "inacabadas" con retoque parcial o inexistente y adelgazamiento bifacial que sitúa a la pieza en una fase muy avanzada de proceso; y "esbozos" con un proceso de trabajo inicial pero lo suficientemente avanzado como para intuir el tipo de pieza que se pretende realizar.

Por otra parte, a la aparición de utillaje específico para la caza, se suman las evidencias faunísticas que se hallan en los niveles solutrenses de la cueva del Higueral-Guardia. Aunque actualmente, dichos restos de fauna se encuentran en fase de estudio, Isabel Cáceres Sánchez y Patrizia Anconetani (Cáceres y Anconetani 1997) realizaron y publicaron un estudio preliminar para los niveles solutrenses en los que analizaron un total de 881 restos pertenecientes principalmente a Cervus elephus, Dama dama, Capra ibex, Capreolus capreolus, Bos primigenius, Oryctolagus cuniculus, y en proporciones menores también documentaron Sus scrofa, Canis lupus y Alectoris rufa (Fig. 8). La estimación que las investigadoras realizan del número mínimo de individuos es de 48 entre los que dominan herbívoros como el conejo, ciervo y cabra. Por otra parte, del estudio tafonómico que se realizó de los restos, se detectaron marcas de carnicería, fracturación intencional sobre hueso fresco. Para la interpretación de las marcas de corte se realizó un estudio binocular y microscópico electrónico de barrido concluyendo la actividad de carnicería (despellejamiento, descuartización, evisceración, descarnación y desarticulación) de estas comunidades con instrumental lítico (Cáceres y Anconetani 1997). 


\subsection{Estudio preliminar de las puntas de pedúnculo y aletas}

Parece no existir unanimidad en el ámbito de la Prehistoria acerca del origen y antigüedad de las puntas como proyectiles aunque tradicionalmente, han sido considerados artefactos característicos del Paleolítico superior. Sin embargo, trabajos más recientes proponen a través de evidencias arqueológicas que el uso de proyectiles comenzaria en poblaciones anteriores entre los 250-40 Ka (O'Brien 1981) rompiendo el consenso inicial entre los investigadores. $\mathrm{Hi}$ pótesis que intentan invalidar estudios experimentales de J. Shea (2006) quien estima que en periodos anteriores al Paleolítico superior las herramientas eran dimensionalmente superiores como para ser efectivos proyectiles.

Más allá de la antigüedad de estos artefactos, conocemos con certeza que las puntas de pedúnculo y aleta solutrenses palmariamente formarian parte de ese conjunto de armas de proyectil que permiten infligir una herida mortal desde una distancia segura. Además de llamativas y eficaces, las puntas de pedúnculo y aletas son sin duda alguna un material característico que permite encuadrar en el Solutrense superior evolucionado a las poblaciones que las elaboraron y habitaron el sur peninsular. Una etapa en la que se registra una paulatina desaparición del retoque plano invasor que caracteriza a este tipo de puntas y una progresiva presencia del retoque abrupto que aparecerá representado en las hojitas de dorso y puntas de muesca (Muñoz 1997).

El conjunto de puntas de pedúnculo y aletas de la cueva del Higueral-Guardia aunque porcentualmente sea una muestra reducida es sin duda un material significativo y consideramos que de haberse dado una actividad clandestina en la cavidad, sería éste tipo de utillaje el más atractivo para su extracción (Fig. 9).

Actualmente conservamos un total de 18 esbozos y puntas de pedúnculo y aletas que aparecieron en el área sondeada número 2. Están realizadas en silex de diversa procedencia y muchas de ellas presentan calentamiento de la materia prima que se traduce en algunas alteraciones térmicas perceptibles a través del craquelado y la presencia de cúpulas, además del aspecto sedoso que presenta el material (pátina térmica). En general, presentan una talla por percusión directa con percutor orgánico y presión muy minuciosa que nos indica el dominio de las técnicas de retoque por presión solutrense por parte de estos grupos. Las puntas varian desde piezas de tamaño medio de unos $40 \mathrm{~mm}$ hasta piezas más pequeñas de $25 \mathrm{~mm}$ y analizadas en conjunto, parece cumplirse la ecuación de Muñoz lbáñez que señala que a medida que aumenta la longitud de la punta también aumenta proporcionalmente las posibilidades de fracturarse en el impacto (Muñoz 1999, 2000; Muñoz y Ripoll 2006). Sin embargo, más allá de las variaciones en tamaño todas parecen perseguir un tipo estándar sobre el que hemos profundizado.

Al observar las piezas se ha planteado la posibilidad de que existiera la idea de reutilizar o reciclar las puntas lo que conduce irremediablemente a dudas terminológicas. Queda un tanto difuso conceptualmente si la recuperación de la funcionalidad de un útil implica reutilización o reciclaje. Unos términos utilizados en ocasiones indistintamente de manera poco acertada. Hosman con el término reciclaje se refiere "al proceso por el que un artefacto que ha pasado por descarte/

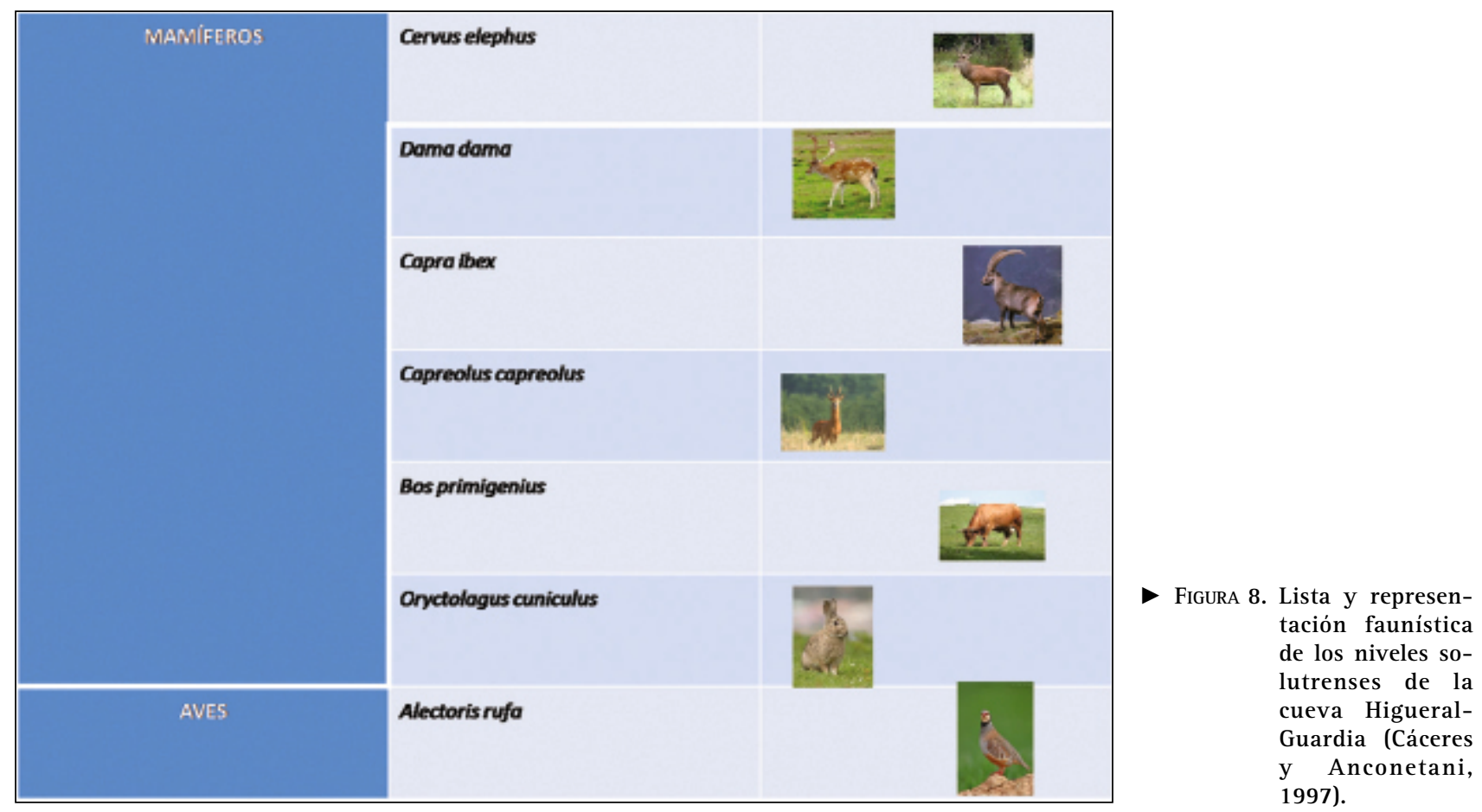




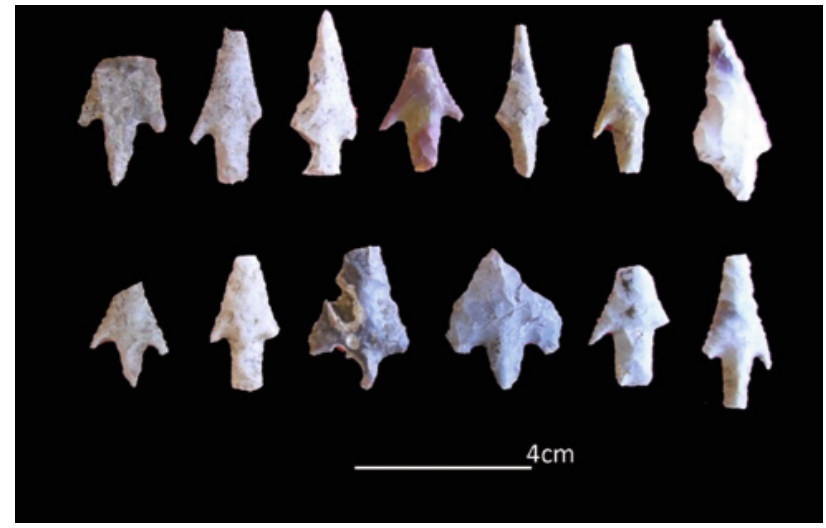

$\Delta$ Figura 9. Trece esbozos y puntas finalizadas de pedúnculo y aletas del total hallado en los niveles solutrenses del sondeo 2 .

abandono, se vuelve a usar" ' . Por su parte, García Cook (Winchkler 2006) define el término reutilización como sustantivo usado con el sentido de "utilización de algo que ya fue usado, en una función laboral distinta" 2. Según ambas definiciones, el hecho de volver a reavivar una punta de pedúnculo y aletas con el objetivo de volverla a usar con el mismo fin no se ajustaría al concepto de reutilización y sí al de reciclaje.

De cualquier modo, más allá de la confusión terminológica matizable se realizó un estudio acerca de la reutilización/reciclaje de las puntas de pedúnculo y aletas a partir de los hallazgos arqueológicos de la cueva del Higueral-Guardia que dio lugar a la realización de un modelo de superposición de contornos de los 18 esbozos y puntas de flecha para establecer ese patrón o punta estándar que consideramos buscaban en la elaboración de este tipo de artefactos. En este modelo los talones encajan perfectamente y la anchura y altura de las piezas suele ser aproximada en todas ellas (Fig. 10). Las líneas que se alejan del conjunto pertenecen a formas inacabadas, fracturadas durante el proceso de talla, pero en algunos casos se aprecia una marcada diferencia dimensional en los principales ejes tipológicos.

La cadena operativa de puntas de pedúnculo y aletas constata que dependiendo del tipo de fractura que se produzca en las puntas, es posible plantearse la posibilidad de reutilizarlas/reciclarlas. Tenemos registrado algún ejemplo de punta posiblemente reutilizada/reciclada, en las que la fractura por uso en el extremo distal permite retallar y usar de nuevo la pieza. Su contorno encaja en el patrón establecido ya que sigue manteniendo su forma pero se reducen sus dimensiones y en ocasiones, aparecen asimetrías en este tipo de piezas tal y como muestran algunas de las piezas del conjunto solutrense de la cueva del Higueral- Guardia (Fig.11). Sin embargo, experimentalmente se constata que las puntas que se rompen mesialmente y por la aleta son abandonadas. El tipo de fractura que se genera en este caso no permite un reaprovechamiento de la pieza (Morrow 1995; Muñoz 1999, 2006; Cheshier y Kelly 2006; Franco 2009). Del mismo modo, encontramos casos en los que solo se conserva el extremo distal de la pieza plenamente elaborado (Fig. 12), lo que indica que la fractura no se produce durante el proceso de elaboración del artefacto sino posteriormente, en su fase de uso. La característica fractura del proyectil por la zona mesial en piezas acabadas se produce por impacto (Fauvelle et al. 2012) y no permite un reaprovechamiento de la pieza. El estudio experimental dirigido por Fauvelle (2012) acerca de lo determinante que resulta el tipo de enmangue en la durabilidad y efectividad de las puntas, viene a demostrar que las fuerzas de resistencia y rotación involucradas en la fase de impacto de un proyectil enmangado originan una fractura característica: la rotura se produce por encima de los tendones que sirven de

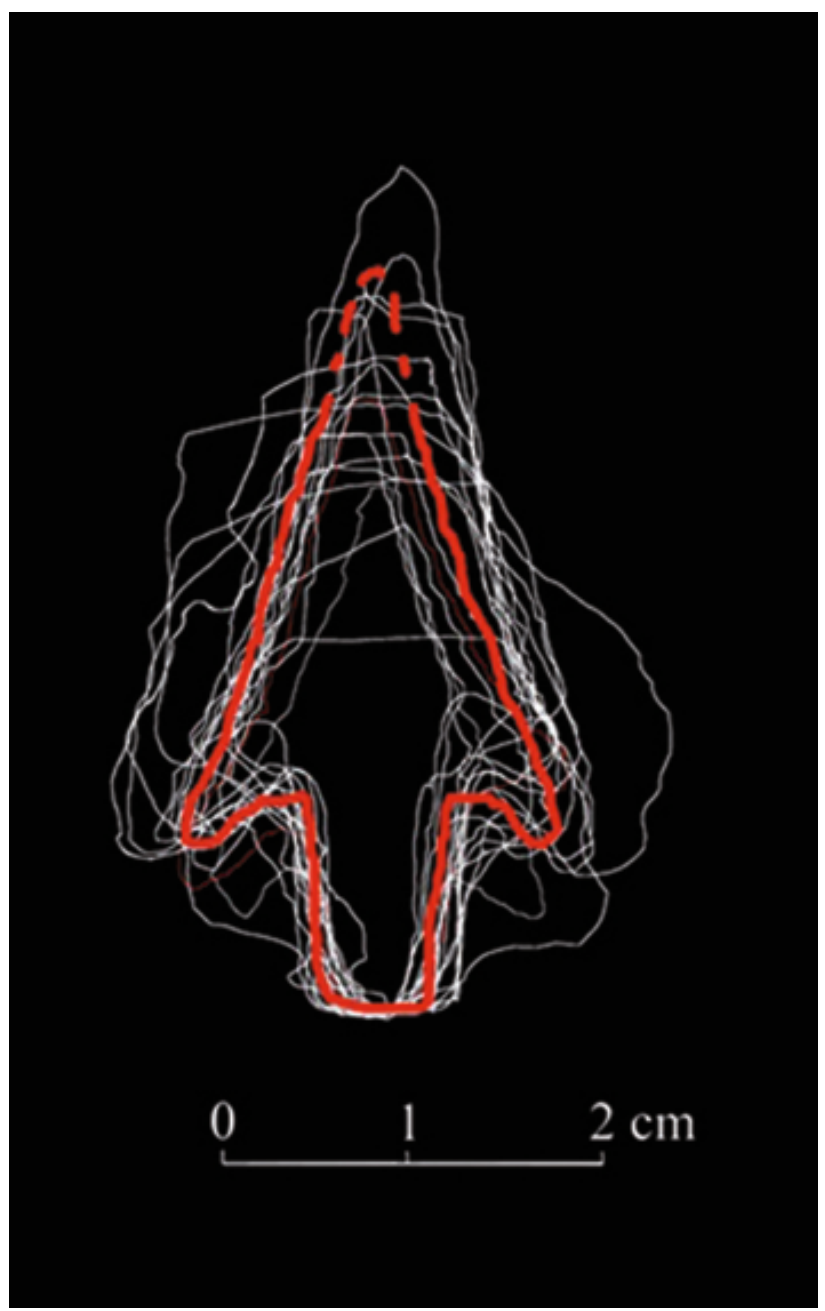

$\Delta$ Figura 10. Modelo de superposición de contornos. En rojo, el posible patrón de las puntas de pedúnculo y aletas.

(1) Cit. por G. Winchkler: Diccionario de uso para la descripción de los objetos líticos, winchkler.com.ar, 2006, p. 221

(2) Cit. por G. Winchkler: Diccionario de uso para la descripción de los objetos liticos, winchkler.com.ar, 2006, p. 234. 
enmangue porque es justo en la zona mesial donde se registra menos resistencia ante la fuerza de impacto.

Entre las puntas arqueológicas encontramos piezas que no han llegado a ser usadas por el tipo de fractura que presenta el artefacto (Maíllo 1999; Muñoz 1999, 2006). Los indicios los encontramos en las propias piezas. La característica fractura por flexión en la zona mesial de la pieza, que suprime todo el extremo distal y parte del cuerpo podría haber sido causado por impacto del proyectil. Sin embargo, observamos a través de su estudio diacrítico, que la pieza está aún en proceso de elaboración. Presenta grandes extracciones que han configurado su contorno y estaba en fase de adelgazamiento (Fig. 12).

En algunas piezas concretas se intuyen diferencias a nivel técnico y tecnológico entre los talladores tal y como ocurre en yacimientos estudiados como Vale Almoinha (MaíIlo 1999) a partir del análisis de los esquemas operativos utilizados en la elaboración de hojas de laurel. En la cueva del Higueral-Guardia, los proyectiles inacabados que no presentan ningún tipo de rotura podrian pertenecer a un grupo de talladores "inexpertos".

La variabilidad morfológica que los materiales de estos "inexpertos" o aprendices, (Fig. 9), no se contradice con la existencia de procesos de reciclaje o reutilización de una parte de los soportes, siempre que los accidentes o fracturas producidas así lo permitan. Los trabajos experimentales que hemos llevado a cabo confirman que una parte importante de los accidentes de talla producidos por talladores expertos (fracturas de extremos distales, proximales o de extremos en las aletas), permiten una reutilización de los soportes accidentados (Figs. 2 y 11). Esta circunstancia queda confirmada por los márgenes de variación dimensional de los contornos analizados en sus diferentes ejes (Fig. 10).

Por último, cabría apuntar a que la existencia de diferentes niveles de tecnicidad, en el seno de los grupos solutrenses que poblaron la zona de las Motillas, confirma la presencia de unidades sociales, con un cierto carácter residencial, y no solo la presencia de altos de caza de carácter logístico en zonas del interior andaluz durante estas fases.

\section{CONCLUSIONES}

La cueva del Higueral-Guardia ha puesto de manifiesto una secuencia geoarqueológica de enorme interés en el contexto interior de la zona de las Béticas occidentales. La existencia de una secuencia, todavía pendiente de un estudio pormenorizado, en la que se documentan niveles de ocupación solutrenses y musterienses sugiere dos cuestiones fundamentales.

Primero, la existencia de relaciones complejas entre áreas costeras e interiores durante el Solutrense meridional. El estudio detallado de estos niveles hubiera podido aclarar el ca- rácter y funcionalidad de la ocupación y con ello facilitar la articulación interpretativa a nivel regional. Desgraciadamente, la alteración de estos niveles, al menos en las zonas sondeadas, limita sensiblemente sus posibilidades en este sentido.

En segundo lugar, la presencia de industria lítica asociada a restos faunísticos con marcas de carnicería y fracturación intencional indica que, al menos en los momentos de ocupación solutrense, la cueva del Higueral-Guardia debió funcionar como lugar de hábitat, donde unas poblaciones especializadas en la caza de herbivoros procesaban sus presas.

Además, la existencia de un hiato ocupacional en la secuencia entre los niveles musterienses y solutrenses, merece una explicación, bien desde un punto de vista geoarqueo-

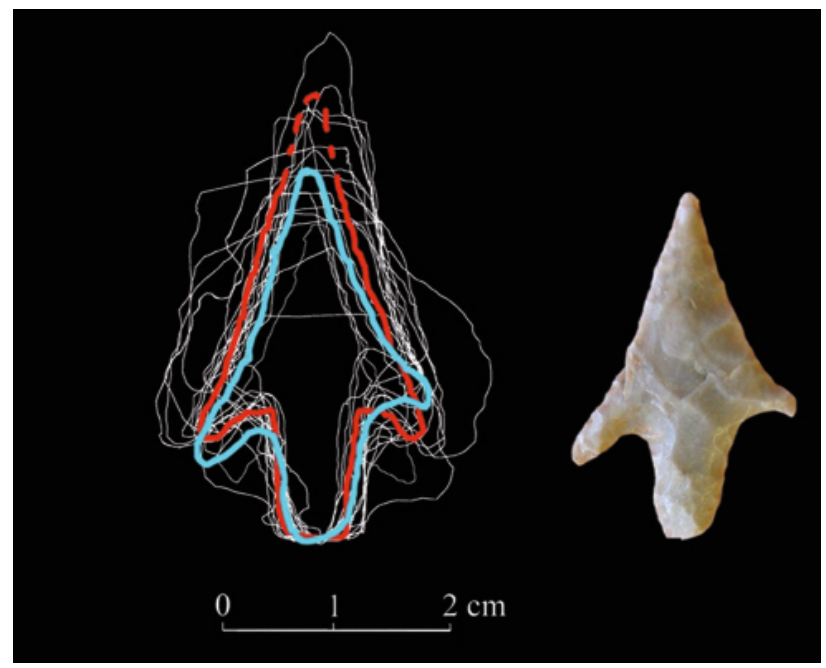

A Figura 11. Posible caso de reutilización/ reciclaje de punta de pedúnculo y aletas.

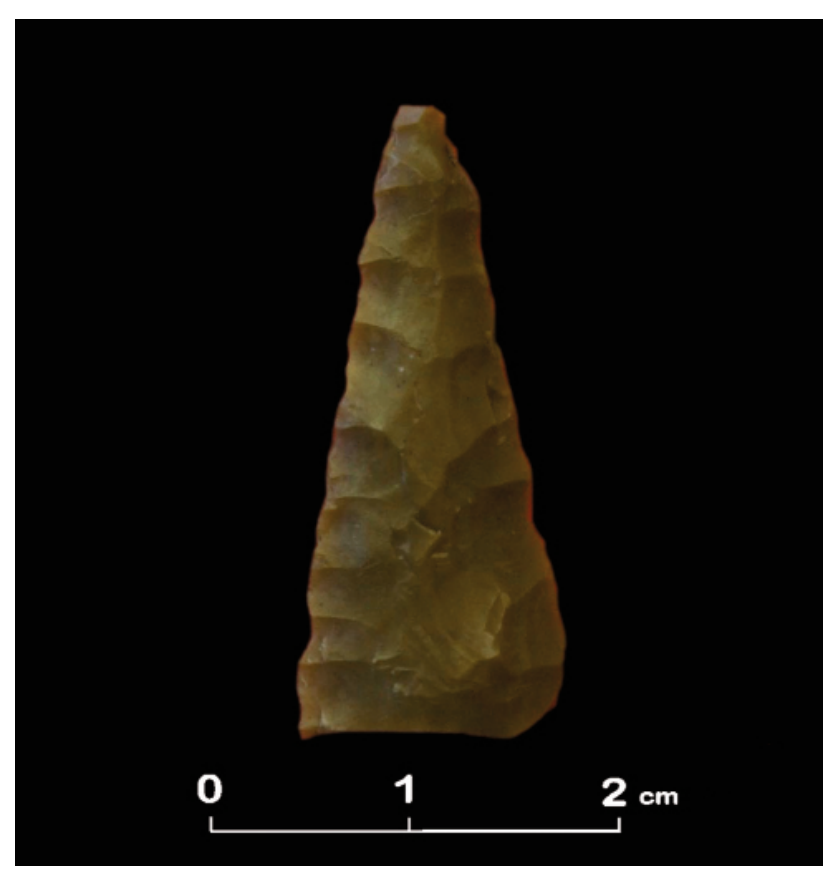

$\Delta$ Figura 12. Extremo distal fracturado por impacto. 


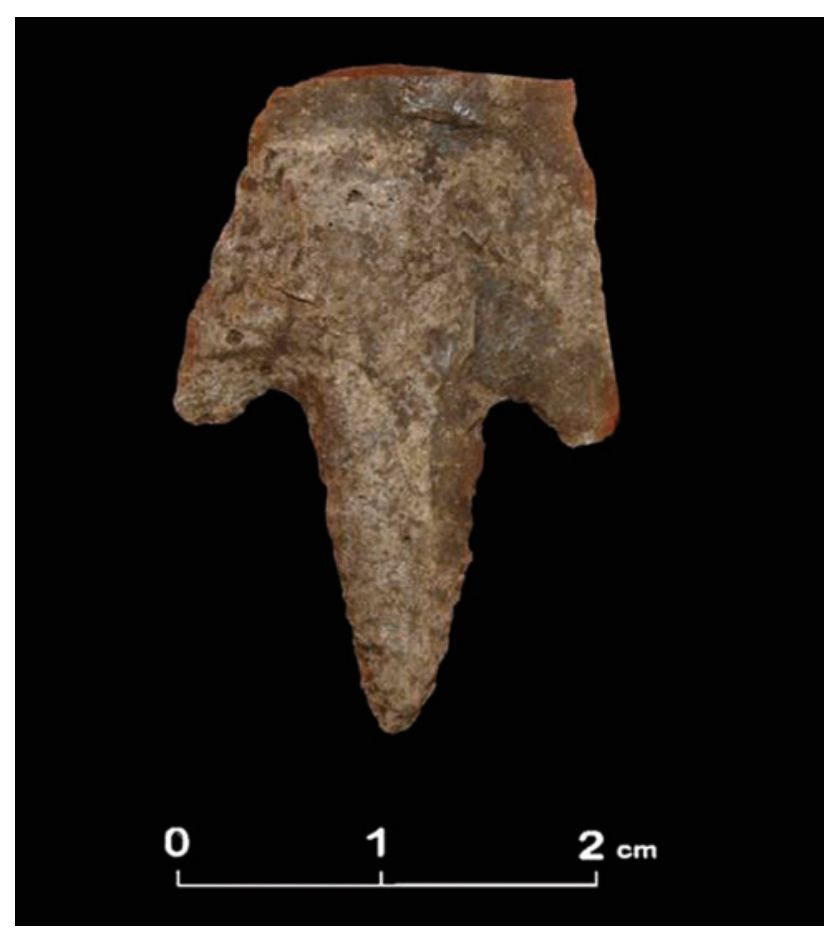

$\Delta$ Figura 13. Punta de pedúnculo y aletas fracturada (flexión con desviación mesial) característica del proceso de elaboración.

lógico, o bien en el contexto de los principales modelos de poblamiento y movilidad de los últimos neandertales y del los grupos de sapiens solutrenses.

La documentación previa y las actuales investigaciones en la cavidad apuntan a un conjunto arqueológico singu- lar. Este hecho motiva la presente actuación en la cueva del Higueral- Guardia sobre el Cerro de Las Motillas para la formación de un inventario y catalogación de los depósitos arqueológicos y su estado de conservación actual. En este sentido, el proyecto KURETES continúa ejecutándose actualmente y esperamos contribuir en un futuro próximo con novedosas e interesantes aportaciones.

En el presente estudio preliminar lamentamos el hecho de solo poder mostrar los materiales abandonados por actividades ajenas a las arqueológicas y planteamos algunas claves metodológicas para el acercamiento a colecciones que han sufrido alteraciones. Sin embargo, a pesar de las variaciones del conjunto, el yacimiento se convierte en un referente fundamental de la ocupación de los primeros humanos modernos en el Sur peninsular.

\section{AGRADECIMIENTOS}

Al proyecto de investigación "KURETES. Primeras ocupaciones humanas, evolución paleoecológica y climática del Cuaternario de las Béticas occidentales (Serranía de Ronda)" financiado por la Consejería de Cultura y Deporte de la Junta de Andalucía. Queremos agradecer además, la colaboración de todos los participantes en los trabajos de campo, y en especial al grupo G40 de Priego de Córdoba. Igualmente al apoyo prestado desde la Consejería de Cultura de la Junta de Andalucía con la aprobación del mencionado proyecto y al ayuntamiento de Cortes de la Frontera (Málaga). •

\section{BIBLIOGRAFÍA}

Arteaga 0., Ramos J. y Roos M. (1998): "La Peña de la Grieta (Porcuna, Jaén). Una nueva visión los cazadores-recolectores del mediodía atlántico-mediterráneo desde la perspectiva de sus modos de vida y trabajo en la Cuenca del Guadalquivir". En J. L. Sanchidrián y M. D. Simón (coords.): Las Culturas del Pleistoceno Superior en Andalucía. Patronato de la Cueva de Nerja. Málaga: 75-109.

BAENA, J. (2011): "Los saqueadores arruinan un yacimiento andaluz". http://www.publico.es/ciencias/397247/los-saqueadores-arruinanun-yacimiento-prehistorico-andaluz

Baena, J., Morgado, A., y Lozano J. A. (en PRensa): "Proyecto KURETES. Primeras ocupaciones humanas, evolución paleoecológica y climática del Cuaternario de las Béticas occidentales (Serranía de Ronda)". Cuadernos de Prehistoria y Arqueología Universidad Autónoma de Madrid.

Barroso Ruiz C., Medina Lara F., Onoratini G. y Joris C. (2006): "Les industries du Paléolithique Supérieur (Protoaurignacien, Gravettien et Solutréen) de la Grotte du Boquete de Zafarraya". En C. Barroso Ruiz y H. de Lumley (dirs.): La Grotte du Boquete de Zafarraya, Málaga, Andalousie. Junta de Andalucia III. Sevilla: 1587-1626.

CÁceres, I. y Anconetanl, P. (1997): "Procesos tafonómicos del nivel solutrense de la cueva de Higueral de Motillas (Cádiz)". Zephyrvs L: 37-52.
Cantalejo, P. (1983): "La Cueva de Malalmuerzo (Moclín, Granada): nueva estación con arte rupestre paleolítico en el área mediterránea". Revista de Antropología y Paleoecología Humana 3: 59-85.

Cantalejo, P., Maura, R., Aranda, A., Espejo, M. M., Ramos, F., Medianero, J., Aranda, A. y DurÁn, J. (2006): La Cueva de Ardales: Arte Prehistórico y ocupación en el Paleolítico Superior. Centro de Publicaciones de la Diputación de Málaga. Málaga.

Córdoba De Oya, B. y Vega Toscano, L. G. (1988): "El Paleolítico de la Sierra del Segura: proyecto de investigación". I Congreso de Historia de Castilla La Mancha 2, (Pueblos y culturas prehistóricas y protohistóricas (1): 79-85.

Cortés SÁnchez, M. (ed.) (2008): El Pirulejo (Priego de Córdoba): Cazadores recolectores del Paleolítico superior en la sierra Subbética. Estudios en homenaje a la profesora María Dolores Asquerino. Antiqvitas 20. Museo Histórico Municipal de Priego. Córdoba.

Cortés SÁnchez, M. y Simón Vallejo, M. D. (2007): "La Pileta (Benaoján, Málaga) cien años después. Aportaciones al conocimiento de su secuencia arqueológica". Sagvntvm 39: 45-62.

Cortés SÁnchez M., Bergadà Zapata M. M., Gibaja BaO J., Jiménez Espejo F., Simón Vallejo M. D. y Riouelme Cantal J. A. (2011): "El Solutrense en la costa de Málaga: contexto paleoambiental y cronocultural". Pyrenae 42 (1): 51-75.

Cheshier, J. y Kelly, R. L. (2006): "Projectile Point Shape and Durability: The Effects of Thickness:Length". American Antiquity 71: 353-363. 
Franco, N. V., Castro A., Cardillo, M. y Charlin J. (2009): "La importancia de las variables morfológicas, métricas y de microdesgaste para evaluar las diferencias en diseños de puntas de proyectil bifaciales pedunculadas: un ejemplo del sur de Patagonia continental". Magallania 37 (1): 99-112.

Fullola J. M., Villaverde V., Sanchidrián J. L., Aura J. E., Fortea, F. J. y SOLER N. (2005): "El Paleolítico Superior Mediterráneo Ibérico". En J. L. Sanchidrián, A. M. Márquez y J. M. Fullola (eds.): IV Simposio de Prehistoria Cueva de Nerja. La Cuenca Mediterránea durante el Paleolítico Superior, 38.000-10.000 años. Patronato de la Cueva de Nerja. Málaga: 192-213.

GieX (1980): El Complejo Motillas-Ramblazo. Jumar 4: 6-16.

Giles, F., SAntiago, A., GutiéRRez, J.M. y MatA, E. (1997): "Las comunidades del Paleolítico Superior en el extremo sur de Andalucia occidental. Estado de la cuestión". En R. de Balbín Behrmann y P. Bueno (eds.): / Congreso de Arqueología Peninsular l: Paleolítico y Epipaleolítico: 383-403.

Giles, F., GutiérRez, J. M., Santiago, A. y MatA, E. (1998): "Avance al estudio sobre el poblamiento del Paleolítico Superior en la cuenca media y alta del Río Guadalete (Cádiz)". En J. L. Sanchidrián y M. D. Simón (coords.): Las Culturas del Pleistoceno Superior en Andalucía. Patronato de la Cueva de Nerja. Málaga: 111-140.

Giles, F., Santiago, A., Aguilera, L., Gutiérrez, J. M. y Finlayson, C. (2000A): "Paleolítico Inferior y Medio en la sierra de Cádiz. Evidencias de grupos de cazadores-recolectores del Pleistoceno Medio y Superior". Almajar1: 8-35.

Giles, F., Gracia, F. J., Santiago, A., GutiérRez, J. M., Mata, E., Agullera, L., Rodriguez, C., Finlayson, C., Piñatel, F. y Barton, N. (2000B): "Nuevas aportaciones al conocimiento de los complejos tecnológicos del Pleistoceno medio y superior del Campo de Gibraltar: Los yacimientos de El Chaparral (Los Barrios) y Guadalquitón-Borondo (San Soque)". Caetaria 3: 13-26

Jennings, R. P., Giles Pacheco, F., Barton, R. N. E., Collcut, S. N., Gale, R., Gleed-OWen, C. P., Gutiérrez López, J. M., Higham, T. F. G., Parker, A. Price, C., Rhodes, E., Santiago Pérez, A., Schwenninger, J. L. y Turner, E. (2009): "New dates and palaeoenvironmental evidence for the Middle to Upper Palaeolithic occupation of Higueral de Valleja Cave, souther Spain". Quaternary Science Reviews 28 (9-10): 830-839.

Maillo Fernández, J. M. (1999): "Esquemas operativos y conocimiento técnico: el caso del yacimiento solutrense del valle Amoinha (Torres Vedras, Portugal)". Espacio, Tiempo y Forma. Serie I, Prehistoria y Arqueología 12: 185-214.

Marqués Merelo, I. y Ruiz, A. C. (1976): "El Solutrense de la Cueva del Tajo de Jorox, Alozaina (Málaga)". Cuadernos de Prehistoria de la Universidad de Granada 1: 77-58.

Morrow, J. E. (1995): "Clovis Projectile Point Manufacture: A Perspective from the Ready/Lincoln Hills Site, Jersey County, Illinois." Midcontinental Journal of Archaelogy 20: 167-191.

MuÑoz, F. J. (1997): "La punta de muesca de tipo mediterráneo: análisis tecnológico y funcional". Trabajos de Prehistoria 54 (1): 99-120.

- (1999): "Algunas consideraciones sobre el inicio de la arqueria prehistórica". Trabajos de Prehistoria 56 (1): 27-40.

- (2000): Las puntas ligeras de proyectil del Solutrense Extracantábrico: análisis tecnomorfológico e implicaciones funcionales. Colección Aula Abierta. UNED. Madrid.
MuÑoZ, F. J. y RIPOLL, S. (2006): "Las primeras evidencias de arcos en Europa: parámetros de diseño y construcción. Zona Arqueológica 7 (1): 463-472.

O'BRIEN, E.M. (1981): "The proyectile capabilities of an Acheulian Handaxe fron Olorgesailie". Current Anthropology 22: 77-79.

RAmos MuÑoz, J., (1998): "La ocupación prehistórica de los medios kársticos de montaña en Andalucia". En J. J. Durán y J. López Martínez (eds.): Karst en Andalucía. Instituto Tecnológico Geominero de España. Madrid: 63-84.

Ripoll López, S. (ED.) (1988): La Cueva de Ambrosio (Vélez Blanco, Almería) y su posición cronoestratigráfica en el Mediterráneo occidental. BAR International Series 462. Archaeopress. 0xford.

SANCHIDRIAN TORTI, J. L. (1997): "Propuesta de la secuencia figurativa en la Cueva de La Pileta". En J. M. Fullola y N. Soler (eds.): El món mediterrani després del Pleniglacial (18.000-12.000 BP). Série Monogràfica 17. Centre d'Investigacions Arqueològiques. Girona: 411-430.

Santiago Vilchez, J. M. (1980a): "Complejo kárstico del Cerro de Las Motillas (Cádiz-Málaga)". SPELEON 25: 47-64.

- (1980b): "Influencia de la estructura en la génesis del karst de las Motillas". Andalucia Subterránea 3. Federación Andaluza de Espeleología. Jerez de la Frontera: 3-13

- (1983): "Notas sobre una prospección arqueológica superficial en el Cerro de las Motillas". SPELEON 26-27: 129-145.

- (1990): Avance al estudio del arte parietal Paleolítico de la Cueva de la Motilla. Zephyrvs, XLIII: 65-76.

TIFFAGom, M. (2006): De la Pierre à I'Homme. Essai sur une paléoanthropologie solutréenne. ERAUL 113. Université de Liège. Lieja.

Tiffagom, M., AuRA, J. E., VILLAVERDE, V. y Fullola, J. M. 2007: "Entre Gravettien et Solutréen en Espagne méditerranéenne : mise en évidence d'une phase intermédiaire à composante mixte?" En M. Almeida, B. Walter y M. J. Neves (eds.): Le Solutréen, 40 ans après Smith '1966. Pré-actes du colloque international de Preuilly-sur-Claise: 28-31.

Toro Moyano I. y Ramos LinazA, M. (1985): "Excavaciones arqueológicas en el yacimiento Solutreogravetiense al aire libre del Pantano de Cubillas. Primeros resultados". Cuadernos de Prehistoria de la Universidad de Granada 10: 9-30.

VALLESPi, E. (1992): "Las industrias achelenses de Andalucia: ordenación y comentarios". Spal 1: 61-78.

Villaverde, V. 1994: Arte Paleolítico de la Cova del Parpalló. Estudio de la colección de plaquetas y cantos grabados y pintados. Servei d'Investigació Prehistòrica. Diputación de Valencia. Valencia.

Villaverde,V., Cardona, J. y Martinez-Valle, R. (2009): "L'art parietal de la grotte Les Meravelles. Vers une caractérisation de l'art paléolithique pré-magdalénien du versant méditerranéen de la Péninsule Ibérique". L'Anthropologie 113 (5): 762-793.

WINCHKLER, G. (2006): Diccionario de uso para la descripción de los objetos líticos, winchkler.com.ar.

ZıเнÃO, J.: (1997): O Paleolitico Superior da Estremadura Portuguesa. 2 vols. Lisboa, ED. Colibri.

Zilinão J., Angeluccl, D., Badal, E., Lucena, A., Martin, I., Martinez, S., Villaverde, V. y ZAPATA, J. (2010): "Dos abrigos del Paleolítico superior en Rambla Perea (Mula, Murcia)". En X. Mangado (ed.): El Paleolítico superior peninsular. Novedades del siglo XXI. Homenaje al profesor Javier Fortea. Monografias del SERP 8. Universitat de Barcelona. Barcelona: 137-148. 
